Under peer review at Meta-Psychology. This is a revised version of the submitted paper. The editorial process, including decision letters and reviews, can be accessed at tinyurl.com/mp-submissions. Anyone can contribute with Open Peer Review through hypothes.is directly on this preprint.

\title{
General claims require generalized effects: A reply to Ruiz et al.'s (2020) 'A systematic and critical response to Pendrous et al. (2020) replication study'
}

\section{Ian Hussey}

\begin{abstract}
Recent evidence suggests replications of psychology studies produce discrepant findings, and that original studies frequently report inflated estimates of effect size. Colloquially speaking, studies 'fail to replicate' at an alarming rate, at least in terms of congruence of finding significant $p$ values. Replies are often then written by the authors of the original studies. This discourse often follows a predictable pattern: highly general claims are made in an original study, the replication produces null results, and the response by the original authors primarily focuses on reasons to disqualify the replication's results from requiring consideration, rather than acknowledging that the original study's finding may not, in fact, be replicable, or that the generality of the original claims may require revision or constraint. I illustrate these points using the example of a recently published trio of an original study, a failed replication, and a response by original authors. I argue that our scientific goals would be better served by efforts to avoid falling into these writing tropes and to instead move the discourse forward and reinforce the behaviours we want to see in our scientific community, such as the conduction of high-quality replication studies. All data and code at osf.io/du8xk.
\end{abstract}

Set in motion in part by the fallout from fiascos such as Bem's (2011) supposed demonstration that individuals could predict the future, the Credibility Revolution in psychology has been characterized in part by the publication of replication studies at a higher rate than previously (Munafò et al., 2017). The large majority of these replications have failed to find the same result as the original studies, leading to somewhat of a replication crisis in psychology (Klein et al., 2018; Open Science Collaboration, 2015). Replication studies, particularly those that failed to find the same result as the original study, have frequently been followed by responses by the authors of the original study.

A common and predictable narrative arc has emerged in these replies: First, original studies tend to make highly general claims based on limited evidence. Next, replication studies are conducted, typically with the same or better levels of rigour as the original studies. In the majority of cases, these replications do not produce the same result as the original study (i.e., in terms of congruence of finding significant $p$ values, see Klein et al., 2018; Open Science Collaboration,
2015). Finally, reply articles by the original authors then frequently focus on differences in methods between the studies, attribute differences in results to these differences in methods, and fail to consider other reasons for the differences in results such as simple false positives or false negatives. In this article, I discuss why this style of reply serves as a barrier to scientific progress, and suggest ways in which we could improve in the future.

In doing so, I highlight one feature of this style of reply that has received less attention to date: whereas original articles tend to make highly general claims without regard to moderators or constraints on generality, replies to failed replications, written by the same authors, often argue that the effect is found only under the highly specific conditions present in the original study. That is, the generality of the claims is frequently not updated in light of the lack of evidence of generality in results across studies. Given that general claims are of greater scientific interest than specific effects in specific methodological preparations, the generality of original claims must be updated and 
constrained in light of new evidence (for related arguments see also de Ruiter, 2018; Simons et al., 2017; Yarkoni, 2019).

I illustrate this point using a trio of recent articles: an original study, replication study and reply by original authors. To briefly recap, Pendrous et al. (2020) recently conducted a replication of Sierra et al.'s (2016) study on acceptance- and metaphor-based interventions to increase pain tolerance on the coldpressor task. Whereas Sierra et al. (2016) found statistically significant evidence for the efficacy of the intervention, Pendrous et al.'s (2020) replication did not. The authors of the original study then responded with a critique of the failed replication in Ruiz, Luciano, and Sierra (2020). This reply focuses primarily on methodological differences between the original and replication study and how these differences may have caused the divergence in results, and which may limit the interpretability of the replications' results. Some of the points that Ruiz et al. (2020) highlighted appear to be correct: Pendrous et al. (2020) claimed methodological superiority when in some cases this does not appear to be the case. I am glad these points were corrected in the record; however, this commentary is more concerned with the elements of Ruiz et al. (2020) that I disagree with.

This article discusses (1) errors or revisions in Ruiz et al.'s (2020) characterization of their original study and their comparison of the original study with the replication, (2) important possible reasons for the differences in results between the two studies that Ruiz et al. (2020) did not consider, and (3) several ways in which Ruiz et al.'s (2020) positions in their reply are incompatible with the generality of their original claims in Sierra et al. (2016).

\section{Revisionism in the importance of methodological features}

Ruiz et al. (2020) is dedicated in large part to documenting differences between Sierra et al. (2016) and Pendrous et al. (2020). Almost universally, the article framed these points in terms of what could be paraphrased as 'failures of Pendrous et al. (2020) to precisely replicate Sierra et al.'s (2016) methods' rather than 'failures of Sierra et al. (2016) to specify the features of their method that are crucial to produce the effect'.

To take one example, Ruiz et al. (2020, p.4) claimed that participants' naivety to Acceptance and Commitment Therapy (Hayes et al., 1999) and/or Relational Frame Theory (Hayes et al., 2001) was an important characteristic of Sierra et al. (2016) that was not methodologically replicated in Pendrous et al. (2020): "Participants in Sierra et al. (2016) were naïve in terms of knowledge about ACT/RFT and experience with the cold pressor task (p. 268). However, $40.5 \%$ and
$23.8 \%$ of the participants in the replication study reported having at least some knowledge of ACT/RFT and previous experience with the cold pressor task, respectively." (p.4)

However, looking up this quote in Sierra et al. (2016) demonstrates that while lack of experience with $\mathrm{ACT} / \mathrm{RFT}$ was reported as a characteristic of the sample, it was explicitly not listed as an exclusion criterion: "Eighty-three undergraduates (42 women; age range $=18$ to $34 ; M=19.47, S D=1.46$ ) attending different courses participated in the experiment. None of them had previous experience with the procedures or the theory (i.e., RFT and ACT) involved in this study. Exclusion criteria were suffering from cardiac and circulatory affections, hypertension, diabetes, epilepsy, chronic pain conditions, or recent wounds (only one participant was excluded because of suffering from arrhythmia) because the experimental task (i.e., coldpressor) might have adverse effects on them."

This is somewhat ironic: while calling for greater systematicity in the reporting of methodological differences between the original and replication studies, Ruiz et al. (2020) have themselves in places mischaracterized the details of Sierra et al. (2016).

In some cases, this involved revising the status of study features. For example, observed characteristics of the sample were implied to be exclusion criteria and/or important moderators of the effect (e.g., experience with ACT/RFT, experience with the cold pressor task). In other places, this involved adding details that were not present in Sierra et al. (2016), for which Pendrous et al. (2020) cannot easily be criticized for not including in their study. For example, Ruiz et al. (2020) stated that "the protocols designed by Sierra et al. contained several crucial pauses to promote the relational activity of the metaphor" (p.13). However, these pauses were not fact noted as 'crucial' in Sierra et al. (2016), they are merely listed by their duration. One or both of these two criticisms can be applied to many other items listed in Ruiz et al.'s (2020) Table 1 "Comparison of the Methodological Characteristics of the Studies": participants' knowledge of ACT/RFT; previous experience with the cold pressor task; method of compensation for participation; brand of apparatus used in the cold pressor task; administration of the experimental procedure (via experimenter or computerized task); whether or not analogical reasoning skills were assessed; and the use of pauses in the acceptance-based protocol.

Implicitly or explicitly, this served to diagnose what Ruiz et al. (2020) argue are issues of methodology in the replication study they could equally have been attributed to failures of communication in the original article about what features of the procedure were important to producing the effect. Methodological 
precision, both in implementation and communication, is the responsibility of all authors.

\section{Implications for the generality of original claims}

Ruiz et al. (2020) considered at length whether Pendrous et al. (2020) qualified as a 'direct' replication, and concluded that it did not meet their criteria for this. However, the utility of this assessment is questionable. First, Ruiz et al.'s (2020) definitions of 'direct' (versus 'systematic') replication were without reference, and are not widely accepted. Indeed, there is wide disagreement about the definitions and utility of terms like 'direct' or 'exact' and the relationship between replicability and claims (Brandt et al., 2014; de Ruiter, 2018; Devezer et al., 2020; Nosek \& Errington, 2019; Simons, 2014; Zwaan et al., 2018). As such, this distinction should not be portrayed as either clear or meaningful. Second, even by Ruiz et al.'s (2020) own definitions, the results of 'systematic' replications can and should be compared with original studies: "If systematic replications find similar effects under different experimental conditions, our confidence in the generality of the findings increases." (p.2). However, no such comparisons were made in the article. Instead, significant attention is paid to the methodological differences between the studies, as discussed in the previous section.

On the one hand, it is important to acknowledge that statements about what methodological features are 'important' or 'crucial' to finding an effect call all too easily represent post hoc conjecture. On the other hand, we could also take these assertions of crucial moderators of the effect at face value. However, doing so represents a radical narrowing of the generality of the claims that were made in the original article. Put simply, if details as small as whether you used the same brand of cold pressor apparatus or the exact wording of the metaphor moderate the effect so strongly, then Sierra et al.'s (2016) highly general conclusions about metaphor in general must also be revised.

Specifically, Sierra et al. (2016) made claims about extremely general phenomena. Its abstract states "metaphor is a tool frequently used in psychotherapy such as Acceptance and Commitment Therapy (ACT), a contextual behavioural model of psychological intervention rooted in an approach to human language and cognition known as Relational Frame Theory (RFT). This experimental analogue study aimed to analyze the effect of two variables in the metaphor effect on promoting psychological flexibility according to RFT." (p.265) Sierra et al.'s (2016) methodology was therefore not intended to capture merely a very specific behavioural effect that could be found under only those conditions. Rather, it was, by the authors' own account, intended to be an analogue of behaviour that occurs in other real-life contexts, and that had implications for behaviour under formally different circumstances (i.e., to capture a specific instance of a more general regularity: Hempel \& Oppenheim, 1948). Indeed, Sierra et al. (2016) close by concluding "according to the results of this study, the ACT therapist from the example of the person in a painful rehabilitation process after surgery should design a metaphor that includes common physical properties with the experienced pain and specify appetitive augmentals to tolerate it."

It is critical to note that Sierra et al. (2016) did not add constraints to this conclusion such as "....as long as the client is naïve to both ACT and RFT, is a student rather than faculty member, and noting that the metaphor will only work if specific pauses are included in its delivery" or a host of other constraints that Ruiz et al. (2020) later argued were actually 'important' or even 'crucial' to find the effect. Such claims about what is essential to producing an effect must be accompanied by revisions to the original claims about the importance of that effect made in Sierra et al. (2016).

If we are to build a cumulative science, this pattern of discourse between original studies, failed replications, and replies by the original authors must be brought full circle and made coherent. Editors, reviewers, and readers must require authors to revisit their claims and make them coherent. Either the effect in question has general implications, in which case it must occur under a wide range of experimental conditions including nonexact replications; or the effect is only detectable under a very narrow range of experimental conditions, in which case implications cannot be general. Simply put, we cannot have our cake and eat it too: general claims require generalized effects (de Ruiter, 2018; Simons et al., 2017; Yarkoni, 2019).

Finally, it should be noted that this claim - that a failure to replicate was driven previously unappreciated variables that are, in fact, 'crucial' to obtain an effect - is so common within the replication literature that it even has a term: an appeal to "hidden moderators" (Simonsohn, 2017). Moreover, it has been the subject of very large-scale replication projects to examine its plausibility as a general explanation for lack of replication. The Many Labs 2 study (Klein et al., 2018) was conducted across a wide range of effects and study contexts ( 28 studies, $n \approx 7000$ per study, data collection in 36 nations), and found very little evidence for hidden moderators. In light of this, claims about key moderators of effects or constraints on their generality must be backed up by data from (preregistered) studies that manipulate these factors rather than post hoc speculation. 


\section{Alternative explanations for differences in results}

Ruiz et al. (2020) raised the question of whether the differences in methods between the studies gave rise to the differences in results. However, they neglected to consider other highly plausible reasons for these differences. The implicit argument was that methodological differences were the only reasons worth discussing. A plausible alternative was not discussed in either Ruiz et al. (2020) or Pendrous et al. (2020): sampling variation, or the possibility of false positives in the original study and/or false negatives in the replication.

Given the divergence in results, it should be acknowledged that it is at least possible that the original study was a false positive. Indeed, this may be more likely than assumed. Sierra et al. (2016) employed two 2X2 ANOVAs and interprets all three of the $p$ values produced by both (i.e., two main effects and an interaction; 6 tests in total). No correction for multiple testing was performed. The false-positive rate for these tests is therefore not $5 \%$, as the alpha level would imply, but $26 \%$ (see Cramer et al., 2016 for mathematical proofs and simulations studies demonstrating this).

Equally, the replication study may have been a false negative. Although Sierra et al. (2016) did not report confidence intervals around the effect sizes they reported, their very low sample size per condition $(n=$ 16) implies that the true effect size, if the true effect is non-zero, could fall within a wide range including being very small. To answer this question quantitatively, I calculated $95 \%$ confidence intervals for the Cohen's $d$ effect sizes reported in Sierra et al. (2016). Means, standard deviations, and sample sizes for percentage change in duration of pain tolerance between pre and post were extracted, and the 'esci' $\mathrm{R}$ package (CalinJageman, 2020) was used to recalculate both Cohen's $d$ point estimates and their $95 \%$ confidence intervals (see Supplementary Materials at osf.io/du8xk). ${ }^{1}$ Confidence interval widths were found to be extremely wide, ranging from $d=1.45$ to 1.56 . This indicated that the data were compatible with a very wide range of true effects, from negative to positive, and very small to very large. As such, it is possible that Pendrous et al. (2020) simply was not powered to detect the effects. Of course, it is worth pointing out that this is not solely a failure of Pendrous et al. (2020): better estimation of the true effect in the original study would arguably have aided all future work through more precise power analyses.
Either way, explicit consideration of the very real possibility of false positives and/or false negatives would have helped counterbalance Ruiz et al.'s (2020) exclusive focus on methodological differences to explain differences in results.

\section{Conclusions}

Ruiz et al. (2020) stated that replication is both "crucial for the progress of science" (p.1) and yet historically it has been difficult to publish such work. Replies by original authors to replications are a salient consequence that serves to either reinforce or punish the probability of doing replication work in the future. If this crucial part of our science is to be reinforced, we must pay more than lip service to its value. Replies to failed replications cannot be divorced from the claims made in original articles. When replies to failed replications invoke moderators or constraints on generality, explicit consideration should be given to the implications for the generality of the original claims.

\section{References}

Bem, D. J. (2011). Feeling the future: Experimental evidence for anomalous retroactive influences on cognition and affect. Journal of Personality and Social Psychology, 100(3), 407-425. https://doi.org/10.1037/a0021524

Brandt, M. J., IJzerman, H., Dijksterhuis, A., Farach, F. J., Geller, J., Giner-Sorolla, R., Grange, J. A., Perugini, M., Spies, J. R., \& van 't Veer, A. (2014). The Replication Recipe: What makes for a convincing replication? Journal of Experimental Social Psychology, 50, 217-224.

https://doi.org/10.1016/j.jesp.2013.10.005

Calin-Jageman, R. J. (2020). esci: Effect sizes and confidence intervals for $R$ and Jamovi. https://github.com/rcalinjageman/esci

Cramer, A. O. J., van Ravenzwaaij, D., Matzke, D. Steingroever, H., Wetzels, R., Grasman, R. P. P. P., Waldorp, L. J., \& Wagenmakers, E.-J. (2016). Hidden multiplicity in exploratory multiway ANOVA: Prevalence and remedies. Psychonomic Bulletin \& Review, 23(2), 640-647. https://doi.org/10.3758/s13423-015-0913-5

de Ruiter, J. P. (2018). The meaning of a claim is its reproducibility. The Behavioral and Brain Sciences, 41, e125.

https://doi.org/10.1017/S0140525X18000602

Devezer, B., Navarro, D. J., Vandekerckhove, J., \& Buzbas, E. O. (2020). The case for formal

\footnotetext{
${ }^{1}$ It should also be noted that the point estimates of Cohen's $d$ reported in Sierra et al. (2016) could not be reproduced from the means and standard deviations they reported. However, it was possible to numerically reproduce the effect sizes reported by applying an incorrect formula for Cohen's $d$ (i.e., by dividing the differences in means by the average standard deviation instead of the pooled standard deviation). In the service of Ruiz et al.'s (2020) call for greater methodological precision in replication studies, it would therefore seem important to also emphasise the need for methodological precision in original studies, including their own.
} 
methodology in scientific reform. Preprint. https://doi.org/10.1101/2020.04.26.048306

Hayes, S. C., Barnes-Holmes, D., \& Roche, B. (2001). Relational frame theory: A post-Skinnerian account of human language and cognition. Kluwer Academic/Plenum Press.

Hayes, S. C., Strosahl, K., \& Wilson, K. G. (1999). Acceptance and Commitment Therapy: An experiential approach to behavior change. Guilford Press.

Hempel, C. G., \& Oppenheim, P. (1948). Studies in the Logic of Explanation. Philosophy of Science, 15(2), 135-175. https://doi.org/10.1086/286983

Klein, R. A., Vianello, M., Hasselman, F., Adams, B. G., Adams, R. B., Alper, S., Aveyard, M., Axt, J. R., Babalola, M. T., Bahník, Š., Batra, R., Berkics, M., Bernstein, M. J., Berry, D. R., Bialobrzeska, O., Binan, E. D., Bocian, K., Brandt, M. J., Busching, R., ... Nosek, B. A. (2018). Many Labs 2: Investigating Variation in Replicability Across Samples and Settings. Advances in Methods and Practices in Psychological Science, 1(4), 443-490. https://doi.org/10.1177/2515245918810225

Munafò, M. R., Nosek, B. A., Bishop, D. V. M., Button, K. S., Chambers, C. D., Percie du Sert, N., Simonsohn, U., Wagenmakers, E.-J., Ware, J. J., \& Ioannidis, J. P. A. (2017). A manifesto for reproducible science. Nature Human Behaviour, 1(1), 0021. https://doi.org/10.1038/s41562-016-0021

Nosek, B. A., \& Errington, T. M. (2019). What is replication? [Preprint]. MetaArXiv. https://doi.org/10.31222/osf.io/u4g6t

Open Science Collaboration. (2015). Estimating the reproducibility of psychological science. Science, $349(6251)$, aac4716.

https://doi.org/10.1126/science.aac4716
Pendrous, R., Hulbert-Williams, L., Hochard, K. D., \& Hulbert-Williams, N. J. (2020). Appetitive augmental functions and common physical properties in a pain-tolerance metaphor: An extended replication. Journal of Contextual Behavioral Science, 16, 17-24.

https://doi.org/10.1016/j.jcbs.2020.02.003

Ruiz, F. J., Luciano, C., \& Sierra, M. A. (2020). A systematic and critical response to Pendrous et al. (2020) replication study. Journal of Contextual Behavioral Science. https://doi.org/10.1016/j.jcbs.2020.04.011

Sierra, M. A., Ruiz, F. J., Flórez, C. L., Hernández, D. R., \& Luciano, C. (2016). The Role of Common Physical Properties and Augmental Functions in Metaphor Effect. International Journal of Psychology, 15.

Simons, D. J. (2014). The value of direct replication. Perspectives on Psychological Science, 9(1), 76-80. https://doi.org/10.1177/1745691613514755

Simons, D. J., Shoda, Y., \& Lindsay, D. S. (2017). Constraints on Generality (COG): A Proposed Addition to All Empirical Papers. Perspectives on Psychological Science, 12(6), 1123-1128. https://doi.org/10.1177/1745691617708630

Simonsohn, U. (2017). "Many Labs" Overestimated The Importance of Hidden Moderators. Data Colada. http://datacolada.org/63

Yarkoni, T. (2019). The Generalizability Crisis. Preprint. https://doi.org/10.31234/osf.io/jqw35

Zwaan, R. A., Etz, A., Lucas, R. E., \& Donnellan, M. B. (2018). Making replication mainstream. The Behavioral and Brain Sciences, 41, 1-50. https://doi.org/10.1017/S0140525X17001972 